\title{
EFICIENCIA DE TRATAMIENTOS PARA EL CONTROL DE HONGOS COMPETIDORES, DURANTE LA PRODUCCIÓN DE CEPA COMERCIAL DE Pleurotus spp.
}

\section{EFFICIENCY OF TREATMENTS FOR THE CONTROL OF COMPETITORS FUNGI, IN THE PRODUCTION OF SEED SPAWN OF Pleurotus spp.}

\author{
García M. Natalia1, " Giraldo B. Dayro² y Castro-Ríos, Katherin² \\ ${ }^{1}$ Universidad de Caldas, Facultad de Ciencias Agropecuarias, Calle 65 No 26 - 10. \\ Manizales, Caldas - Colombia \\ ${ }^{2}$ Servicio Nacional de Aprendizaje, SENA, Grupo de Investigación en Biotecnología, \\ Seguridad Alimentaria y Nutricional (BIOSAN), Km 10 Vía al Magdalena. Manizales, Caldas \\ - Colombia.
}

Recibido 21 de Octubre 2015; aceptado 30 de Marzo de 2016

\section{RESUMEN}

Los hongos comestibles del género Pleurotus spp., se caracterizan por crecer fácilmente en diversos sustratos y condiciones climáticas, sin embargo esto incide también en el crecimiento de hongos competidores que disminuyen la productividad del cultivo. Por tal razón, se propuso evaluar la eficiencia de tratamientos físicos y químicos en el control de hongos contaminantes del cultivo, en diversas etapas del proceso de obtención de cepa comercial de Pleurotus spp. Se evaluó la temperatura de esterilización del sustrato y medio de cultivo y la concentración del hipoclorito en la desinfección de los carpóforos. Luego de evaluar los tratamientos, se obtuvo que el aumento en la temperatura de esterilización del medio de cultivo, la combinación de esterilización e irradiación UV aplicado al sustrato y una desinfección con hipoclorito de sodio al 0.01 
$\%$, fueron las condiciones adecuadas para controlar los hongos contaminantes durante los ensayos realizados.

*Autor a quien debe dirigirse la correspondencia. Giraldo B. Dayro" e-mail: dagiraldo@misena.edu.co.
Palabras clave: Desinfección, Esterilización, Hongo comestible, Hongo ostra, Semilla comercial.

\begin{abstract}
The edible mushrooms of the gender Pleurotus spp., are characterized by easily grow in various substrates and climatic conditions, however this also affects the growth of competitors fungi that reduces the productivity of the crop. For this reason, it was proposed to evaluate the efficiency of physical and chemical treatments in the control of fungi contaminants from the mushroom crop, at various stages of the process to obtaining commercial strain of Pleurotus spp. Assessed the sterilization temperature of the substrate and the culture medium and the concentration of sodium hypochlorite for the disinfection of the carpophores. After evaluating the treatments, it is obtained that the increase in the temperature of sterilization of the culture medium, the combination of sterilization and UV radiation applied to the substrate and disinfection with sodium hypochlorite at $0.01 \%$, were the appropriate conditions to control pollutants fungi during the tests performed.
\end{abstract}

Keywords: Disinfection, Mushroom, Oyster mushroom, Seed spawn, Sterilization.

\section{INTRODUCCIÓN}

Pleurotus spp., es un hongo de pudrición blanca de gran relevancia en el mundo por su papel en la degradación de material biológico; presenta un importante aporte nutricional debido a la calidad de la proteína, la amplia distribución de aminoácidos esenciales y no esenciales; el contenido de carbohidratos, fibra dietética y minerales (Goyal, Grewal, \& Goyal, 2006; Mukhopadhyay \& Guha, 2015), y además posee la ventaja de poder ser cultivado en una amplia variedad de sustratos. Diferentes estudios reportan la bioconversión de residuos agroindustriales e industriales, empleando este hongo 
@,LIMENTECH CIENCIA Y TECNOLOGÍA ALIMENTARIA ISSN 1692-7125. Volumen 14 No. 1, p. 27 - 37 año 2016 Facultad de Ingenierías y Arquitectura

Universidad de Pamplona

comestible en sustratos como: desechos de cultivos de maíz, ajo, caña, aceite de oliva, arroz y fibras de planta; además de sobrantes de la industria maderera, papelera y de biodiesel (Bernardi, Minotto, \& Nascimento, 2013; da Luz, Paes, Torres, Nunes, da Silva, Mantovani, et al., 2013; Lechner \& Monaldi, 2011; Mandeel, AlLaith, \& Mohamed, 2005; Vargas, Hoyos, \& Mosquera, 2012; Varnero, Quiroz, \& Álvarez, 2010).

Los estudios sobre hongos comestibles realizados en Colombia, se han centrado en el aprovechamiento de residuos agrícolas, como los subproductos del cultivo e industrialización del café (pulpa, cisco, película plateada, aserrín de tallos de café y borra de café (Rodríguez \& Jaramillo, 2004). Además, se han evaluado residuos de cultivos de papa, plátano, caña de azúcar, maíz, arvejas, frutas y flores (Castaño, Goyes, Albarracín, \& López, 2012; López-Rodríguez, HernándezCorredor, Suárez-Franco, \& Borrero, 2008; Montoya Barreto, Franco, \& López, 2009; Motato R., Mejía G., \& León P., 2006; Rivera Omen, Martínez Mamián, \& Morales Velasco, 2013; Vargas, Hoyos, \& Mosquera, 2012). Lo anterior se debe a que Colombia presenta una vocación predominantemente agropecuaria, que representa un $6.3 \%$ del PIB del país (World Bank, 2016), teniendo por tanto, un alto impacto en la generación de subproductos en dicho sector, debido a lo cual, el cultivo de hongos se ha desarrollado como una alternativa de aprovechamiento y generación de valor agregado a estos residuos.

Uno de los problemas que se presenta en el cultivo de hongos comestibles en el país, es la competencia por el sustrato con otros microorganismos, lo cual impacta negativamente la producción del micelio del hongo o cepa comercial, la productividad del cultivo y las características físicas del cuerpo fructífero; esto genera importantes pérdidas económicas a los productores $(\mathrm{N}$. Mazumder, Rathaiah, \& Gogoi, 2005; Sobieralski, Siwulski, KommonŻelazowska, Błaszczyk, Sas-Golak, \& Frużyńska-Jóźwiak, 2012). Diversos estudios relacionados con Pleurotus spp. han identificado la presencia de microorganismos competidores como: Aspergillus flavus var. cotumneris, $A$. niger, Alternaria alternata, Penicillium janthinellum, Penicillium spp, Rhizopus stotoniter, Bacillus brevis, Pseudomonas tolaasii y Trichoderma spp, siendo este último, una especie de hongo que inhibe el crecimiento del micelio de Pleurotus spp. (Blaszczyk, Siwulski, Sobieralski, \& Fruzynska-Jozwiak, 2013; Mazumder, Rathaia, \& Gogoi, 2005; Sobieralski, Siwulski, Kommon-Żelazowska, Błaszczyk, Sas-Golak, \& Frużyńska-Jóźwiak, 2012; Sobieralski, Siwulski, Komon-Żelazowska, Błaszczyk, Górski, Spiżewski, et al., 2012). 
Se han utilizado alternativas biológicas para el control de este tipo de contaminantes en el cultivo de Pleurotus spp, entre ellas el aceite esencial de Cymbopogon citratus L., que demostró una inhibición de los hongos Aspergillus flavus, A. fumigatus, A. niger, Alternaria alternata, Penicillium citrinum, Curvularia lunata y Trichoderma harzianum, a concentraciones mayores a 1500 ppm en ensayos in-vitro (Mahanta, Chutia, Bordoloi, Pathak, Adhikary, \& Sarma, 2007). En otro estudio fueron evaluados ocho extractos en etanol (Azadiracta indica, Allium sativum, Artemesia indica, Urtica dioeca, Lycopersicon esculentum, Datura stramonium, Mentha spicata y Juglans regia) en pruebas in-vitro, obteniéndose hasta $52 \%$ de inhibición de Trichoderma harzianum con el extracto de Juglans regia; sin embargo estos extractos también generaron un efecto inhibitorio en el micelio de Pleurotus spp. (Shah, Nasreen, \& Munshi, 2011). El extracto en metanol de la resina de Asafoetida oleogum a concentración entre 0.65 y 1.25 $\mu \mathrm{g} / \mathrm{L}$, también permitió detener la proliferación de Trichoderma harzianum, en un trabajo in-vitro con Pleurotus eryngii (Angelini, Pagiotti, Venanzoni, \& Granetti, 2009).

También, se han evaluado fungicidas como Carbendazim, Bitertanol, Hexaconazol, Captan y Mancozeb en el control de Trichoderma harzianum. El fungicida que presentó mejores resultados fue Carbendazim con una inhibición del $91 \%$; sin embargo también generó una inhibición de $25 \%$ en Pleurotus sajor-caju, aunque fue menos agresivo que otros fungicidas evaluados, en donde la inhibición del hongo comestible fue de hasta $87 \%$ (Shah, Nasreen, \& Kousar, 2013). Otros tratamientos físicos como la esterilización a $121{ }^{\circ} \mathrm{C}$, agua caliente a $60{ }^{\circ} \mathrm{C}$ y agua alcalina con inmersión mayor a 36 h, han mostrado que limitan el crecimiento Trichoderma spp. al ser evaluados en el sustrato para el cultivo de Pleurotus ostreatus (Colavolpe, Mejia, \& Alberto, 2014; Contreras, Sokolov, Mejía, \& Sánchez, 2004; Oseni, Dlamini, Earnshaw, \& Masarirambi, 2012).

Como puede evidenciarse en los trabajos expuestos, aún quedan interrogantes sobre el control más adecuado de hongos competidores en el proceso de obtención de Pleurotus spp., que se ajusten a las necesidades y condiciones ambientales particulares. Por este motivo en el presente trabajo se evaluó la eficiencia de tratamientos físicos, para el control de hongos competidores, durante la obtención de cepa comercial de Pleurotus spp. 
@LIMENTECH CIENCIA Y TECNOLOGÍA ALIMENTARIA

ISSN 1692-7125. Volumen 14 No. 1, p. 27 - 37 año 2016

Facultad de Ingenierías y Arquitectura

Universidad de Pamplona

\section{MATERIALES Y MÉTODOS}

\section{Localización y obtención de carpóforos de Pleurotus spp.}

El estudio fue realizado en la "Planta didáctica de procesamiento de hongos comestibles", del SENA Regional Caldas, ubicada en Latitud: 5o 4' 01,88 Norte, Longitud 31'05,45 Oeste, temperatura promedio de $18^{\circ} \mathrm{C}$, altitud de $2160 \mathrm{msnm}$. En este lugar se obtuvieron los carpóforos de Pleurotus spp., los cuales fueron sembrados en sustrato compuesto por pulpa de café y tratado térmicamente a 80 oC durante 8 horas. Después de 4 meses, se procedió a la cosecha y selección de carpóforos, utilizados posteriormente en la obtención de cepa comercial. El cuerpo fructífero fue lavado con agua potable y desinfectado con hipoclorito de sodio a diferentes concentraciones de $(0.01,0.02 \mathrm{y}$ $0.03 \%$ ), durante 10 minutos. Luego se realizó un triple enjuague con agua destilada estéril.

\section{Extensión de micelio en caja de petri.}

Los carpóforos seleccionados fueron sembrados, cortados con una cuchilla estéril en cuadros de $1 \mathrm{~cm}^{2}$ y depositados en el medio de cultivo Agar PDA (Oxoid ${ }^{\circledR}$ ), como se observa en la figura 1 a. Posteriormente las cajas de petri con el carpóforo, fueron incubadas a $25 \stackrel{\circ}{\circ}$ (Incubadora Memmert), hasta que el micelio cubriera la caja de petri en su totalidad (Figura 1b).
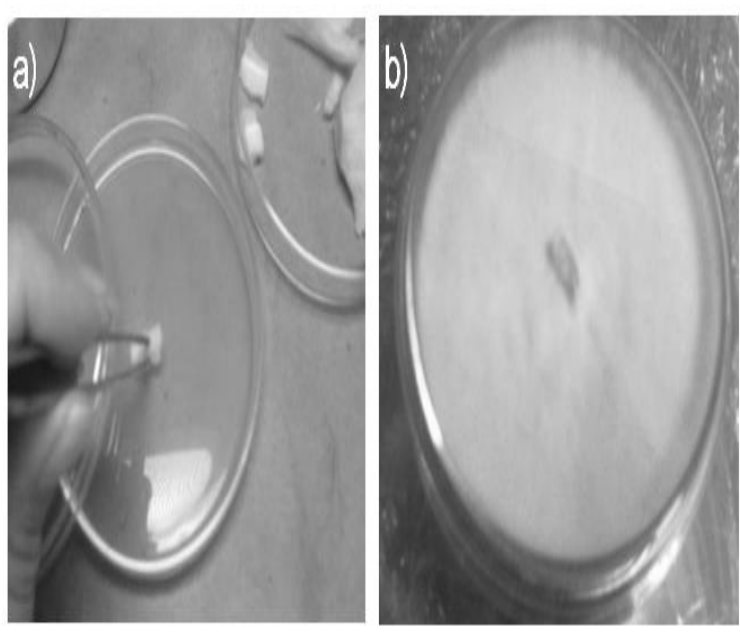

Figura 1. Obtención de micelio de Pleurotus spp.

En esta etapa del proceso, se evaluaron las condiciones de esterilización en la preparación del medio de cultivo (Agar PDA). Para esto se seleccionaron dos temperaturas de esterilización $\left(121^{\circ} \mathrm{C}\right.$ y $134^{\circ} \mathrm{C}$ ), con variables constantes de $15 \mathrm{lb}$ de presión y un tiempo estándar de 15 minutos. La esterilización se realizó en un equipo autoclave de 24 I (All American). Posteriormente, se procedió a la obtención de cepa comercial.

\section{Obtención de cepa comercial}

Se seleccionó $1 \mathrm{~cm}^{2}$ del micelio de Pleurotus spp., y se depositó en bolsas de polipropileno, con $1 \mathrm{Kg}$ de sustrato de semilla de trigo (Figura 2b), el cual fue previamente lavado e hidratado durante 48 h. Se evaluaron dos procedimientos de esterilización: en el primer procedimiento el sustrato fue sometido a un tratamiento térmico a $121^{\circ} \mathrm{C}$, y en el segundo, el 
(a)LIMENTECH CIENCIA Y TECNOLOGÍA ALIMENTARIA ISSN 1692-7125. Volumen 14 No. 1, p. 27 - 37 año 2016 Facultad de Ingenierías y Arquitectura

Universidad de Pamplona

tratamiento térmico a $121 \stackrel{\circ}{\circ} \mathrm{C}$ fue combinado con el tratamiento físico UV, ambos a $15 \mathrm{lb}$ de presión y un tiempo estándar de 15 minutos, en un equipo autoclave de 24 I (All American). Las bolsas esterilizadas e inoculadas fueron incubadas a $26^{\circ} \mathrm{C}$ en una cámara aséptica oscura, hasta que el micelio cubriera completamente la bolsa (Figura 2b). Finalmente se evaluó la contaminación de la cepa comercial de Pleurotus spp. en las bolsas (presencia de micelio diferente al del hongo Pleurotus spp.).
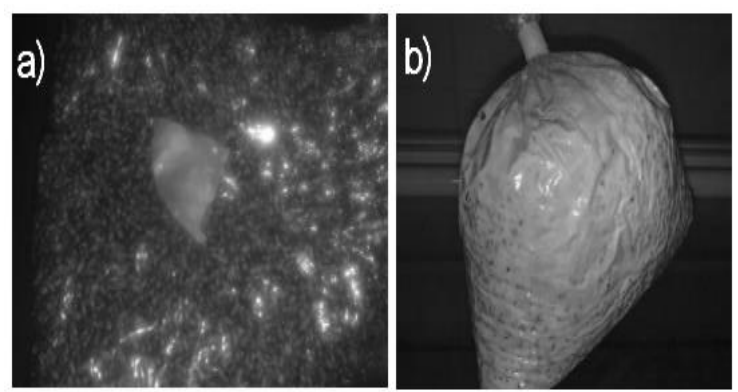

Figura 2. Obtención de cepa comercial de Pleurotus spp.

\section{Análisis estadístico}

\section{RESULTADOS Y DISCUSIÓN}

\section{Desinfección de carpóforos}

Las concentraciones de hipoclorito de sodio empleadas en la desinfección de los carpóforos de Pleurotus spp., presentaron diferencias significativas $(p<0.05)$, respecto a la contaminación de la cepa comercial. El análisis de los datos que se observa en la tabla 2, evidencia diferencias estadísticas
Para el análisis de datos se realizó estadística descriptiva y diseño experimental aleatorizado, con un nivel de significancia del $5 \%$. Las muestras fueron analizadas por duplicado, a través de un análisis de varianza, empleando el programa Statgraphics ${ }^{\circledR}$ plus. Los factores y niveles estudiados se muestran en la tabla 1, la variable de respuesta para los ensayos, fue el porcentaje de contaminación de la cepa comercial de Pleurotus spp. (Presencia de micelio diferente al del hongo comestible).

Tabla 1. Factores y niveles empleados en el estudio.

\begin{tabular}{|c|c|c|}
\hline Ensayos & Factores & Magnitudes \\
\hline $\begin{array}{c}\text { Desinfección de } \\
\text { carpóforos. }\end{array}$ & $\begin{array}{c}\text { Concentración } \\
\text { de hipoclorito } \\
\text { de sodio. }\end{array}$ & $\begin{array}{c}0.01,0.02 \text { y } \\
0.03 \%\end{array}$ \\
\hline $\begin{array}{c}\text { Esterilización del } \\
\text { medio de cultivo. }\end{array}$ & $\begin{array}{c}\text { Temperatura } \\
\text { de } \\
\text { esterilización. }\end{array}$ & $121^{\circ} \mathrm{C}$ y \\
$134^{\circ} \mathrm{C}$ \\
\hline $\begin{array}{c}\text { Esterilización del } \\
\text { sustrato. }\end{array}$ & $\begin{array}{c}\text { Temperatura } \\
\text { de } \\
\text { esterilización } \\
\text { y tratamiento } \\
\text { físico. }\end{array}$ & $\begin{array}{c}121^{\circ} \mathrm{C} \text { y } \\
121^{\circ} \mathrm{C}+\end{array}$ \\
$\mathrm{UV}$
\end{tabular}

entre las concentraciones de hipoclorito de sodio $0.01 \%-0.03 \%$ y $0.02 \%-0.03 \%$, pero inexistencia de diferencia estadística entre $0.01 \%$ y $0.02 \%$. La tabla 2 muestra un aumento en la contaminación de la cepa comercial de Pleurotus spp. con el incremento en la concentración de hipoclorito de sodio, esto puede estar 
relacionado con un efecto directo del desinfectante sobre el crecimiento del micelio de hongo. Por lo tanto, mayores concentraciones de hipoclorito de sodio afectan de forma más efectiva a Pleurotus spp. lo cual reduce la competencia por el sustrato, permitiendo que los hongos patógenos lo contaminen fácilmente.

Lo anterior sólo se evidencia con hipoclorito, como ocurre en el presente estudio, también se ha observado en otros trabajos desarrollados. Un resultado similar fue reportado empleando otro desinfectante químico: peróxido de hidrógeno $\left(\mathrm{H}_{2} \mathrm{O}_{2}\right)$, en donde a mayor concentración del desinfectante se presenta una mayor afectación del crecimiento micelial del hongo, según las características genéticas de cada cepa (Zharare, Kabanda, \& Poku, 2010). Extractos botánicos en etanol también mostraron efectos inhibitorios en el desarrollo del micelio de Pleurotus spp., con inhibiciones entre 17 y $61 \%$, según el extracto empleado (Shah, Nasreen, \& Munshi, 2011). Este tipo de inhibiciones también han ocurrido, con valores por encima del $63 \%$ al utilizar fungicidas como Bitertanol, Hexaconazol y Mancozeb (Shah, Nasreen, \& Kousar, 2013).

Tabla 2. Efecto de la desinfección de carpóforos con hipoclorito de sodio en la contaminación de la cepa comercial de Pleurotus spp.

\begin{tabular}{|c|c|}
\hline $\begin{array}{c}\text { Concentración de } \\
\text { hipoclorito de } \\
\text { sodio. }\end{array}$ & $\begin{array}{c}\text { Contaminación de } \\
\text { cepa comercial de } \\
\text { Pleurotus spp. }\end{array}$ \\
\hline $0.01 \%$ & $5 \%^{\mathrm{a}}$ \\
\hline $0.02 \%$ & $30 \%^{\mathrm{ab}}$ \\
\hline $0.03 \%$ & $60 \%^{\mathrm{c}}$ \\
\hline $\begin{array}{l}\text { Superíndices con diferente letra indican } \\
\text { diferencia significativa. }\end{array}$
\end{tabular}

\section{Esterilización del medio de cultivo}

Se presentó diferencia estadística significativa $\quad(p<0.05) \quad$ entre las temperaturas de esterilización en el medio de cultivo y los valores de contaminación de cepa comercial de Pleurotus spp. Como se evidencia en la tabla 3, la contaminación del sustrato a $121^{\circ} \mathrm{C}$ fue de $50 \%$, mientras que a $134^{\circ} \mathrm{C}$ solamente fue del $10 \%$, esto indica que el aumento de temperatura en la esterilización tiene un efecto positivo al evitar la contaminación de la cepa comercial. A mayor temperatura aumentan los valores de letalidad del proceso, por lo tanto hay una rápida disminución de los microorganismos contaminantes (Brennan \& Grandison, 2012). Sin embargo, también es posible la degradación de los nutrientes del medio de cultivo y un aumento en los costos, al emplear mayor temperatura.

Tabla 3. Efecto de la esterilización del medio de cultivo en la contaminación de la cepa comercial de Pleurotus spp.

\begin{tabular}{|c|c|}
\hline $\begin{array}{l}\text { Temperatura de } \\
\text { esterilización }\end{array}$ & $\begin{array}{l}\text { Contaminación de } \\
\text { cepa comercial de } \\
\text { Pleurotus spp. }\end{array}$ \\
\hline $121^{\circ} \mathrm{C}$ & $50 \%^{\mathrm{a}}$ \\
\hline $134{ }^{\circ} \mathrm{C}$ & $10 \%^{\mathrm{b}}$ \\
\hline
\end{tabular}


(a)LIMENTECH CIENCIA Y TECNOLOGÍA ALIMENTARIA

ISSN 1692-7125. Volumen 14 No. 1, p. 27 - 37 año 2016

Facultad de Ingenierías y Arquitectura

Universidad de Pamplona

\section{Esterilización del sustrato en combinación con UV}

En la tabla 4, se aprecia una considerable disminución en el porcentaje de contaminación del $10 \%$ al $60 \%$, cuando se emplea esterilización a $121{ }^{\circ} \mathrm{C}$ en combinación con UV, evidenciando una diferencia estadística significativa $(p<0.05)$ entre los tratamientos comparados. EI resultado sugiere que la combinación del tratamiento térmico y el UV presentan una mayor eficiencia en la reducción de los hongos competidores, en comparación con el tratamiento térmico únicamente. Además, podría indicar que existe una sinergia entre la temperatura y la radiación UV, lo que permite que el efecto físico generado por la esterilización se una al generado por el UV al interior de la célula de los hongos contaminantes, influenciando así la actividad y descomposición de los hongos (Duguay \& Klironomos, 2000).

Tabla 4. Efecto de la esterilización del sustrato en la contaminación de la cepa comercial de Pleurotus spp.

\begin{tabular}{|c|c|}
\hline $\begin{array}{c}\text { Temperatura de } \\
\text { esterilización }\end{array}$ & $\begin{array}{c}\text { Contaminación de } \\
\text { cepa comercial de } \\
\text { Pleurotus spp. }\end{array}$ \\
\hline $121^{\circ} \mathrm{C}$ & $60 \%^{\mathrm{a}}$ \\
\hline $\begin{array}{c}121^{\circ} \mathrm{C}+\mathrm{UV}(1 \\
\text { hora })\end{array}$ & $10 \%{ }^{\mathrm{b}}$ \\
\hline
\end{tabular}

Superíndices con diferente letra indican diferencia significativa.

\section{CONCLUSIONES}

Es importante el control de los microorganismos contaminantes durante las diferentes etapas de producción del cultivo de Pleurotus spp., dado que impactan negativamente el proceso, al afectar el crecimiento del micelio y posterior formación del cuerpo fructífero.

Luego de realizar la evaluación de los tratamientos que inciden en la eficiencia de cepa comercial de Pleurotus spp., pudo determinarse que las condiciones adecuadas para disminuir la contaminación de hongos competidores fueron las siguientes: concentraciones de hipoclorito de sodio de $0.01 \%$ en la desinfección de carpóforos, temperatura de esterilización del medio de cultivo a $134^{\circ} \mathrm{C}$ y esterilización del sustrato a $121^{\circ} \mathrm{C}+\mathrm{UV}$.

En el presente estudio, se observaron alternativas viables para disminuir la incidencia de hongos competidores durante el proceso de obtención de cepa o semilla comercial de Pleurotus spp.: una baja concentración de hipoclorito de sodio que permita inhibir los contaminantes, sin afectar el desarrollo del hongo comestible; la correcta esterilización del medio de 
(a)LIMENTECH CIENCIA Y TECNOLOGÍA ALIMENTARIA

ISSN 1692-7125. Volumen 14 No. 1, p. 27 - 37 año 2016

Facultad de Ingenierías y Arquitectura

Universidad de Pamplona

cultivo; y la esterilización del sustrato en

combinación con irradiación UV.

\section{REFERENCIAS BIBLIOGRÁFICAS}

Angelini, P., Pagiotti, R., Venanzoni, R., \& Granetti, B. Antifungal and allelopathic effects of Asafoetida against Trichoderma harzianum and Pleurotus spp. (2009). Allelopathy Journal. 23(2): 357-368

Bernardi, E., Minotto, E., \& Nascimento, J. S. d. Evaluation of growth and production of Pleurotus sp. in sterilized substrates. (2013). Arquivos do Instituto Biológico. 80: 318-324.

Blaszczyk, L., Siwulski, M., Sobieralski, K., \& Fruzynska-Jozwiak, D. Diversity of Trichoderma spp. causing Pleurotus green mould diseases in Central Europe. (2013). Folia Microbiol (Praha). 58(4): 325-333.

Brennan, J. G., \& Grandison, A. S. Food Processing Handbook (Vol. 1). Weinheim (Alemania): Wiley- $\mathrm{VCH}$ Verlag \& Co, 2012.

Castaño, N. L., Goyes, P., Albarracín, L. C., \& López, F. J. Uso del bagazo enriquecido con el hongo Pleurotus ostreatus, en dietas para bovinos estabulados en ceba. (2012). Biotecnología en el Sector Agropecuario y Agroindustrial. 10: 25-33.
Colavolpe, M. B., Mejia, S. J., \& Alberto, E. Efficiency of treatments for controlling Trichoderma spp during spawning in cultivation of lignicolous mushrooms. (2014). Braz J Microbiol. 45(4): 12631270.

Contreras, E. P., Sokolov, M., Mejía, G., \& Sánchez, J. E. Soaking of substrate in alkaline water as a pretreatment for the cultivation of Pleurotus ostreatus. (2004). The Journal of Horticultural Science and Biotechnology. 79(2): 234240.

Da Luz, J. M. R., Paes, S. A., Torres, D. P., Nunes, M. D., da Silva, J. S., Mantovani, H. C., \& Kasuya, M. C. M. Production of edible mushroom and degradation of antinutritional factors in jatropha biodiesel residues. (2013). LWT - Food Science and Technology. 50(2): 575580.

Duguay, K. J., \& Klironomos, J. N. Direct and indirect effects of enhanced UV-B radiation on the decomposing and competitive abilities of saprobic fungi. (2000). Applied Soil Ecology. 14(2): 157164. 
(a)LIMENTECH CIENCIA Y TECNOLOGÍA ALIMENTARIA

ISSN 1692-7125. Volumen 14 No. 1, p. 27 - 37 año 2016

Facultad de Ingenierías y Arquitectura

Universidad de Pamplona

Goyal, R., Grewal, R. B., \& Goyal, R. K.

Nutritional Attributes of Agaricus

contamination of Pleurotus. (2005).

Indian Phytopathology. 58(1): 84-88.

bisporus and Pleurotus sajor caju

Mushrooms. (2006). Nutrition and

Health. 18(2): 179-184.

Lechner, B. E., \& Monaldi, S. Utilization of garlic and maize wastes supplemented with olive mill waste water for Pleurotus ostreatus cultivation. (2011). Revista mexicana de micología. 34: 17-22.

López-Rodríguez, C., HernándezCorredor, R., Suárez-Franco, C., \& Borrero, M. Evaluación del crecimiento y producción de Pleurotus ostreatus sobre diferentes residuos agroindustriales del departamento de Cundinamarca. (2008). Universitas Scientiarum. 13: 128-137.

Mahanta, J. J., Chutia, M., Bordoloi, M., Pathak, M. G., Adhikary, R. K., \& Sarma, T. C. Cymbopogon citratus L. essential oil as a potential antifungal agent against key weed moulds of Pleurotus spp. spawns. (2007). Flavour and Fragrance Journal. 22(6): 525-530.

Mandeel, Q. A., Al-Laith, A. A., \& Mohamed, S. A. Cultivation of oyster mushrooms (Pleurotus spp.) on various lignocellulosic wastes. (2005). World Journal of Microbiology and Biotechnology. 21(4): 601-607.

Mazumder, N., Rathaia, Y., \& Gogoi, R. Seasonal variation in microbial

Montoya Barreto, S., Franco, G. M. R., \& López, L. A. T. Rendimientos en el cultivo de los hongos Pleurotus ostreatus, Pleurotus pulmonarius utilizando como sustratos diversos residuos agroindustriales. (2009). Revista de Investigaciones(14):16-26.

Motato R., K. E., Mejía G., A. I., \& León P., Á. Evaluación de los residuos agroindustriales de plátano (Musa paradisíaca) y aserrín de abarco (Cariniana piriformes) como sustratos para el cultivo del hongo Pleurotus djamor. (2006). Vitae. 13: 24-29.

Mukhopadhyay, R., \& Guha, A. K. A comprehensive analysis of the nutritional quality of edible mushroom Pleurotus sajor-caju grown in deproteinized whey medium. (2015). LWT - Food Science and Technology. 61(2): 339-345.

N. Mazumder, Rathaiah, Y., \& Gogoi, R. Seasonal variation in microbial contamination of Pleurotus ostreatus spawn. (2005). Indian Phytopathology. 58(1): 84-88.

Oseni, T., Dlamini, S. O., Earnshaw, D. M., \& Masarirambi, M. Effect of Substrate Pre-treatment Methods on Oyster Mushroom (Pleurotus ostreatus) Production. (2012). Int. J. Agric. Biol. 14(2): 251-255. 
Rivera Omen, R. L., Martínez Mamián, C. A., \& Morales Velasco, S. Evaluación de residuos agrícolas como sustrato para la producción de Pleurotus ostreatus. (2013). Luna Azul. (37):89-100.

Rojas, C. L., Cajiao, A. Cárdenas, R. Aislamiento de hongos en las diferentes etapas del beneficio de café cultivado y comercializado en Toledo, Norte de Santander, (2015), @limentech, Ciencia y Tecnología Alimentaria. Vol. 13. № 2. P. $96-107$.

Rodríguez, N., \& Jaramillo, C. Cultivo de hongos comestibles del género Pleurotus sobre residuos agrícolas de la zona cafetera. (2004). Chinchiná: Cenicafe.

Shah, S., Nasreen, S., \& Kousar, S. Efficacy of Fungicides against Trichoderma spp. Causing Green Mold Disease of Oyster Mushroom (Pleurotus sajor-caju). (2013). Research Journal of Microbiology. 8(1): 13-24.

Shah, S., Nasreen, S., \& Munshi, N.A Evaluation of Some Botanicals in Controlling Green Mold (Trichoderma harzianum) Disease in Oyster Mushroom Cultivation. (2011). International Journal of Botany. 7(3): 209-215.

Sobieralski, K., Siwulski, M., KommonŻelazowska, M., Błaszczyk, L., SasGolak, I., \& Frużyńska-Jóźwiak, D. Impact of Trichoderma Pleurotum and $T$.
Pleuroticola Isolates on Yielding of Pleurotus Ostreatus (FR.) (2012). Kumm. Journal of Plant Protection Research. 52(1):

Sobieralski, K., Siwulski, M., KomonŻelazowska, M., Błaszczyk, L., Górski, R., Spiżewski, T., \& Sas-Golak, I. Evaluation of the Growth of Trichoderma Pleurotum and Trichoderma Pleuroticola Isolates and Their Biotic Interaction with Pleurotus Sp. (2012). Journal of Plant Protection Research. 52(2): 235-239

Vargas, P. S., Hoyos, J. L., \& Mosquera, S. A. Uso de hojarasca de roble y bagazo de caña en la producción de Pleurotus ostreatus. (2012). Biotecnología en el Sector Agropecuario y Agroindustrial. 10(1): 136-145.

Varnero, M. T., Quiroz, M. S., \& Álvarez, C. H. Utilización de Residuos Forestales Lignocelulósicos para Producción del Hongo Ostra (Pleurotus ostreatus). (2010). Información tecnológica. 21(2): 13-20.

World Bank. Agricultura, valor agregado (\% del PIB) / 2016. Disponible en: http://datos.bancomundial.org/indicador /NV.AGR.TOTL.ZS. Consultado: Junio 10 del 2016.

Zharare, G. E., Kabanda, S. M., \& Poku, J. Z. Effects of temperature and hydrogen peroxide on mycelial growth of eight Pleurotus strains. (2010). Scientia Horticulturae. 125(2): 95-102. 